\title{
Azimuthal angle dependence of di-jet production in unpolarized hadron scattering
}

\author{
Zhun Lu and Ivan Schmidt \\ Departamento de Física, Universidad Técnica Federico Santa María, Casilla 110-V, Valparaíso, Chile \\ and Center of Subatomic Physics, Valparaíso, Chile
}

\begin{abstract}
We study the azimuthal angular dependence of back-to-back di-jet production in unpolarized hadron scattering $H_{A}+H_{B} \rightarrow J_{1}+J_{2}+X$, arising from the product of two Boer-Mulders functions, which describe the transverse spin distribution of quarks inside an unpolarized hadron. We find that when the di-jet is of two identical quarks $\left(J_{q}+J_{q}\right)$ or a quark-antiquark pair $\left(J_{q}+J_{\bar{q}}\right)$, there is a $\cos \delta \phi$ angular dependence of the di-jet, with $\delta \phi=\phi_{1}-\phi_{2}$, and $\phi_{1}$ and $\phi_{2}$ are the azimuthal angles of the two individual jets. In the case of $J_{q}+J_{q}$ production, we find that there is a color factor enhancement in the gluonic cross-section, compared with the result from the standard generalized parton model. We estimate the $\cos \delta \phi$ asymmetry of di-jet production at RHIC, showing that the color factor enhancement in the angular dependent of $J_{q}+J_{q}$ production will reverse the sign of the asymmetry.
\end{abstract}

PACS numbers: 12.38.Bx, 13.85.Ni, 13.88.+e

\section{INTRODUCTION}

The role of transverse momentum dependent (TMD) distributions [1, 2] in high energy physics has received a great deal of attection recently. This major interest in TMD distributions relies on the belief that they are responsible for various azimuthal asymmetries observed in different single particle inclusive processes involving at least two hadrons. Examples are the single transverse spin asymmetry (SSA) measured in semi-inclusive deeply inelastic scattering (SIDIS) 3, 4] and inclusive pion production in hadron collision [5, 6], as well as large $\cos 2 \phi$ anomalous asymmetry in the Drell-Yan process [7, 8, 9]. It is found that leading-twist time-reversal-odd ( $T$-odd) distributions play an essential role in these asymmetries, i.e. the Sivers function [10] can account for the SSA, while the Boer-Mulders function [2, 11] can explain the large $\cos 2 \phi$ anomaly. A key ingredient of $T$-odd distributions is the path-ordered exponential contained in their definition, the so called Wilson line or gauge-link [12, 13, 14] due to initial-state or final-state interactions [15] between the active partons and the spectator system. For example, final state interactions are related to a future pointing Wilson line (denoted as $\mathcal{U}^{[+]}$) for $T$-odd distributions, connected to the SSA in SIDIS processes. On the other hand, the study of Drell-Yan processes shows that initial-state interactions give rise to a past-pointing Wilson line $\left(\mathcal{U}^{[-]}\right)$, which predicts a minus sign difference of the Sivers functions [13, 14, 15] and correspondingly the SSA in the Drell-Yan process compared to the SIDIS process. Based on these studies, TMD Factorization in SIDIS, Drell-Yan and $e^{+} e^{-}$annihilation has been established [16, 17], within the allowance of a sign reversal for $T$-odd functions in different processes.

Recently further studies [18, 19, 20, 21, 22, 23, 24, 25] have been performed on back to back di-jet or di-hadron production in hadron-hadron collisions. Unlike in SIDIS and Drell-Yan processes, in which there is only either finalstate interactions or initial state interactions, in hadron-hadron collisions both initial-state and final-state interactions contribute to the process. The result is that there is a more complicated form of the gauge-link for TMD distribution functions in hadron-hadron collisions than in SIDIS or Drell-Yan processes. For example the loop structure $\mathcal{U}^{[\square]}=$ $\mathcal{U}^{[-]^{\dagger}} \mathcal{U}^{[+]}$will appear in the corresponding gauge-link [18]. Further studies [20] show that in hadron-hadron collisions the form of the gauge-link depends even on the hard partonic process. The impact of these nontrivial gauge-links on phenomenology, especially on SSA, has been studied in back-to-back hadron pair or di-jet production [19, 21, 26], and photon-jet productions [27]. In Refs. [23, 28], the authors show that in second order of gluon exchange from initialand final-state interaction, the standard TMD factorization is violated in back-to-back hadron (jet) pair production, i.e., the TMD distributions need to be modified in a process-dependent way (See also Ref. [29], where the universalbreaking part of the TMD distributions has been given).

As shown in Refs. 19, 21], a consequence of nontrivial gauge-links is that in hadron-hadron scattering the parton distribution (and fragmentation) functions are convoluted with gluonic pole cross-sections rather than the standard basic parton cross-sections. Compared to the basic parton cross-sections, there is a gluonic pole factor $C_{G}$ along with the gluonic cross-section, which arises from the $T$-odd effect contributed by the multiple initial- and final-state interactions mediated by gluon exchanges. The complete gluonic pole cross-section encountered in SSA in hadronhadron scattering have been given in Ref. [19, 21] for hadron pair, di-jet and hadron-jet production, and in Ref. [27] for photon-jet production. In this paper, we will study the azimuthal angle dependence of back-to-back di-jet produced in unpolarized hadron-hadron scattering. Like the SSA in di-jet production, where one incident hadron is transversely polarized, there is also interesting phenomenology in the case of di-jet production in unpolarized hadron-hadron 


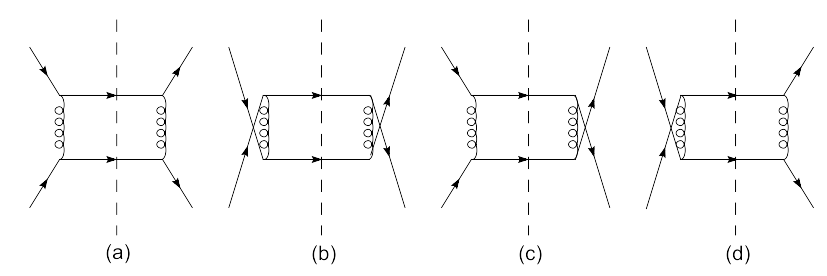

FIG. 1: partonic diagrams contributing to di-jet production $H_{A}+H_{B} \rightarrow J_{q}+J_{q}+X$.

scattering. First, one realizes that T-odd TMD distributions, especially the Boer-Mulders function, produces also azimuthal asymmetries in unpolarized hadron scattering, similar to the case of the cos $2 \phi$ asymmetry in unpolarized Drell-Yan processes. A recent example is the analysis of that asymmetry of photon-jet production in unpolarized hadron scattering, given in Ref. [30]. Therefore the study will provide more knowledge on the TMD distributions which play a role in the process. Second, it is interesting to investigate the effect of multiple initial- and finalstate interactions on the cross-section of di-jet production in unpolarized hadron scattering, as has been done in single transversely polarized hadron scattering. In unpolarized hadron scattering the azimuthal asymmetry can be produced by two $T$-odd distributions, which will make the gluonic pole factor more complicated. Therefore a quantitative study of the effect of multiple initial- and final-state interaction on unpolarized hadron scattering will shed light on the QCD dynamics that is present in azimuthal asymmetries.

\section{ANGULAR DEPENDENCE OF DI-JET PRODUCTION IN UNPOLARIZED HADRON SCATTERING}

The process we study is

$$
H_{A}\left(P_{A}\right)+H_{B}\left(P_{B}\right) \rightarrow J_{1}\left(P_{1}\right)+J_{2}\left(P_{2}\right)+X
$$

where the two incident hadrons $H_{A}$ and $H_{B}$ are unpolarized. The momenta of these initial hadrons are denoted by $P_{A}$ and $P_{A}$, and those of the di-jet by $P_{1}$ and $P_{2}$. We are interested in the kinematical region where the transverse momenta of the di-jet, $P_{1 \perp}$ and $P_{2 \perp}$, have similar size and are almost back to back

$$
\boldsymbol{P}_{1 \perp} \approx-\boldsymbol{P}_{2 \perp},\left|\boldsymbol{P}_{1 \perp}\right| \approx\left|\boldsymbol{P}_{2 \perp}\right| \approx P_{\perp}
$$

and where the total transverse momentum of the di-jet $\boldsymbol{q}_{\perp}=\boldsymbol{P}_{1 \perp}+\boldsymbol{P}_{2 \perp}$ is much smaller than $\boldsymbol{P}_{\perp}: q_{\perp}=\left|\boldsymbol{q}_{\perp}\right| \ll P_{\perp}$. Therefore the process is sensitive to the intrinsic transverse momenta of the partons inside the hadron. We label the azimuthal angles of $\boldsymbol{P}_{1 \perp}$ and $P_{2}$ as $\phi_{1}$ and $\phi_{2}$, respectively. We also define $\delta \phi=\pi-\left(\phi_{2}-\phi_{1}\right)$, which describes the imbalance of the di-jet from a back to back configuration.

The cross-section of di-jet production can be calculated from the general form

$$
d \sigma=\frac{1}{2 s}|\mathcal{M}|^{2} \frac{d^{3} P_{1}}{(2 \pi)^{3} 2 E_{P_{1}}} \frac{d^{3} P_{2}}{(2 \pi)^{3} 2 E_{P_{2}}},
$$

where the amplitude square is expressed as a convolution of the parton-parton to di-jet hard amplitudes and the correlation functions [19]:

$$
\begin{aligned}
|\mathcal{M}|^{2} & =\int d x_{1} d^{2} \boldsymbol{k}_{1 \perp} d x_{2} d^{2} \boldsymbol{k}_{2 \perp}(2 \pi)^{4} \\
& \times \delta^{4}\left(k_{1}+k_{2}-P_{1}-P_{2}\right) \operatorname{Tr}\left\{\Phi\left(x_{1}, \boldsymbol{k}_{1 \perp}^{2}\right) \Phi\left(x_{2}, \boldsymbol{k}_{2 \perp}^{2}\right)\right. \\
& \left.\times H\left(k_{1}, k_{2}, P_{1}, P_{2}\right) H^{\star}\left(k_{1}, k_{2}, P_{1}, P_{2}\right)\right\} .
\end{aligned}
$$

In the above equation we denote the momenta of the initial partons as $k_{1}$ and $k_{2}$, respectively.

The Mandelstam variables of the partonic process are defined as

$$
\begin{aligned}
& \hat{s}=\left(k_{1}+k_{2}\right)^{2}, \\
& \hat{t}=\left(k_{1}-P_{1}\right)^{2}, \\
& \hat{u}=\left(k_{1}-P_{2}\right)^{2},
\end{aligned}
$$




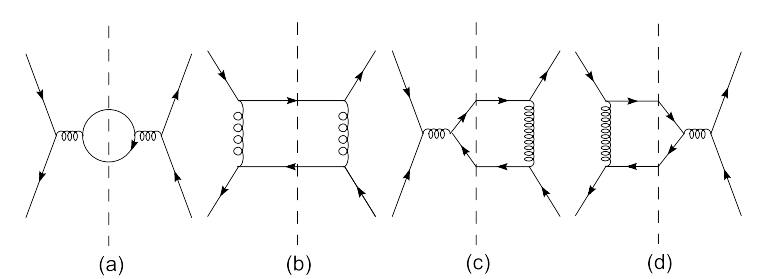

FIG. 2: Partonic diagrams contributing to di-jet production $H_{A}+H_{B} \rightarrow J_{q}+J_{\bar{q}}+X$ through $q \bar{q} \rightarrow q \bar{q}$.

which satisfy the relation

$$
\frac{\hat{t}}{\hat{s}}=-y=-\frac{1}{e^{\left(\eta_{1}-\eta_{2}\right)}+1}, \quad \frac{\hat{u}}{\hat{s}}=-(1-y),
$$

where $\eta_{1 / 2}$ are the pseudorapidities of the jets.

Since we will only consider unpolarized scattering, the correlation matrix element (Soft part) for the unpolarized hadron can be simply decomposed as [1, 2]

$$
\Phi\left(P, x, \boldsymbol{k}_{\perp}^{2}\right)=\frac{1}{2}\left[f_{1}\left(x, \boldsymbol{k}_{\perp}^{2}\right) \not P+\frac{h_{1}^{\perp}\left(x, \boldsymbol{k}_{\perp}^{2}\right)}{M} k_{\perp}^{\mu} P^{\nu} \sigma_{\mu \nu}\right],
$$

where $f_{1}\left(x, \boldsymbol{k}_{\perp}^{2}\right)$ is the unpolarized TMD quark distribution, and $h_{1}^{\perp}\left(x, \boldsymbol{k}_{\perp}^{2}\right)$ is the Boer-Mulders function describing the transverse spin distribution of a quark in an unpolarized hadron [2].

In di-jet production, the transverse momenta of the particles in the di-jet are measured in order to probe the corresponding parton transverse momentum, and in this sense the azimuthal angle dependence of the di-jet is of most interest. The cross-section of unpolarized di-jet production contains both an azimuthal angle independent part and an azimuthal angle dependent part. For the azimuthal angle independent part, all possible partonic scattering subprocesses can contribute to the process, while only the unpolarized TMD distributions $f_{1}\left(x, p_{T}^{2}\right)$ does. On the other hand, for the azimuthal angle dependent part, the Boer-Mulders function $h_{1}^{\perp}$ is involved, and since $h_{1}^{\perp}$ is a chiral-odd TMD function, only partonic processes that flip the helicity of the quark will contribute. The possible partonic processes are $q\left(k_{1}\right)+q\left(k_{2}\right) \rightarrow q\left(P_{1}\right)+q\left(P_{2}\right)$ and $q\left(k_{1}\right)+\bar{q}\left(k_{2}\right) \rightarrow q\left(P_{1}\right)+\bar{q}\left(P_{2}\right)\left(\right.$ or $\left.q^{\prime}\left(P_{1}\right)+\bar{q}^{\prime}\left(P_{2}\right)\right)$. Since the contribution of $h_{1}^{\perp g}$ is power suppressed, we will not consider the gluon contribution to the azimuthal angle asymmetry in unpolarized di-jet production. Therefore we will specifically study the azimuthal angular dependence of di-jet in unpolarized $H_{A}+H_{B} \rightarrow J_{q}+J_{q}+X$ and in $H_{A}+H_{B} \rightarrow J_{q}+J_{\bar{q}}+X$. However, since quark-channels make only a small contribution to jet rates (unless $P_{\perp}$ is very large) this suppression might be compensated by the abundance of gluons and large scattering cross sections for gluons.

\section{A. Process $H_{A}+H_{B} \rightarrow J_{q}+J_{q}+X$}

For the process $H_{A}+H_{B} \rightarrow J_{q}+J_{q}+X$, the corresponding partonic subprocess is $q q \rightarrow q q$, and the lowest-order cut diagrams are shown in Fig. 1. According to Eq. (4), one can therefore arrive at the angular independent cross-section given by:
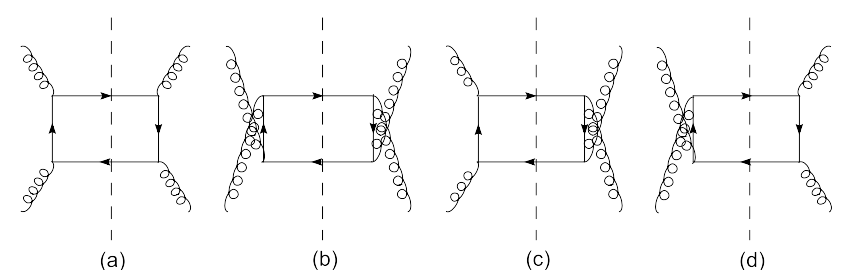

FIG. 3: Partonic diagrams contributing to di-jet production $H_{A}+H_{B} \rightarrow J_{q}+J_{\bar{q}}+X$ through $g g \rightarrow q \bar{q}$. 


$$
\begin{aligned}
\frac{d \sigma^{O}\left[J_{q} J_{q}\right]}{d^{2} \boldsymbol{P}_{1 \perp} d^{2} \boldsymbol{P}_{2 \perp} d \eta_{1} d \eta_{2}} & =\frac{\alpha_{s}^{2}}{s \hat{s}}\left\{\frac{N^{2}-1}{2 N^{2}}\left(\frac{1+(1-y)^{2}}{y^{2}}+\frac{1+y^{2}}{(1-y)^{2}}\right)-\frac{\left(N^{2}-1\right)}{N^{3}} \frac{1}{y(1-y)}\right\} \\
& \times \sum_{q} e_{q}^{2} \int d^{2} \boldsymbol{k}_{1 \perp} d^{2} \boldsymbol{k}_{2 \perp} \delta^{2}\left(\boldsymbol{k}_{1 \perp}+\boldsymbol{k}_{2 \perp}-\boldsymbol{P}_{1 \perp}-\boldsymbol{P}_{2 \perp}\right) f_{1}^{q}\left(x_{1}, \boldsymbol{k}_{1 \perp}^{2}\right) f_{1}^{q}\left(x_{2}, \boldsymbol{k}_{2 \perp}^{2}\right),
\end{aligned}
$$

with $\hat{s}=P_{\perp}^{2} /(y(1-y))$. The momentum fractions $x_{1}$ and $x_{2}$ can be expressed as

$$
x_{1}=\frac{P_{\perp}}{\sqrt{s}}\left(e^{\eta_{1}}+e^{\eta_{2}}\right), \quad x_{2}=\frac{P_{\perp}}{\sqrt{s}}\left(e^{-\eta_{1}}+e^{-\eta_{2}}\right) .
$$

After integrating over $P_{2 \perp}$ and $\phi_{1}$, this leads to the expression

$$
\begin{aligned}
& \frac{d \sigma^{O}\left[J_{q} J_{q}\right]}{d P_{\perp}^{2} d \eta_{1} d \eta_{2}}=\frac{\pi}{s \hat{s}}\left\{\frac{N^{2}-1}{2 N^{2}}\left(\frac{1+(1-y)^{2}}{y^{2}}+\frac{1+y^{2}}{(1-y)^{2}}\right)\right. \\
& \left.-\frac{\left(N^{2}-1\right)}{N^{3}} \frac{1}{y(1-y)}\right\} \sum_{q} e_{q}^{2} f_{1}^{q}\left(x_{1}\right) f_{1}^{q}\left(x_{2}\right),
\end{aligned}
$$

and the parton transverse momentum integration is de-convoluted by the $\delta$-function. In the above equation we have used $d^{2} \boldsymbol{P}_{1 \perp}=\frac{1}{2} d \boldsymbol{P}_{1 \perp}^{2} d \phi_{1} \approx \frac{1}{2} d P_{\perp}^{2} d \phi_{1}$.

Our purpose is to study the azimuthal angle dependence of unpolarized di-jet production. We recall that similar studies of angular dependence have been performed for the case of the unpolarized Drell-Yan process [11]. Due to the correlation between the quark transverse spin and transverse momentum, the product of two Boer-Mulders functions $h_{1}^{\perp} \times h_{1}^{\perp}$ can give rise to large $\cos 2 \phi$ angular asymmetries of dilepton production in unpolarized Drell-Yan processes [11, 31, 32]. One can expect that the same mechanism can produce non-zero angular dependence of di-jets in unpolarized hadronic scattering. Actually a recent study [30] on photon-jet hadronic production shows that $h_{1}^{\perp}$ gives an angular dependence very similar to the $\cos 2 \phi$ asymmetry in Drell-Yan processes. We will follow this line to study the azimuthal angle dependence of unpolarized di-jet production.

Since $h_{1}^{\perp}$ is a chiral-odd function which flips the helicity between the incoming and ongoing quarks, among the diagrams shown in Fig. 1, only Fig. 1, and Fig. 1 can couple with $h_{1}^{\perp}$ and consequently contribute to the angular dependence of $J_{q}+J_{q}$ production. Thus according to Eqs. (4) and (9), the contribution of $h_{1}^{\perp}$ to the cross-section of unpolarized $J_{q}+J_{q}$ can be expressed as

$$
\begin{aligned}
& \frac{d \sigma^{A}\left[J_{q} J_{q}\right]}{d^{2} \boldsymbol{P}_{1 \perp} d^{2} \boldsymbol{P}_{2 \perp} d \eta_{1} d \eta_{2}}=\frac{4 C_{q q} \alpha_{s}^{2}}{M^{2} s \boldsymbol{P}_{\perp}^{4}}\{y(1-y)\} \sum_{q} e_{q}^{2} \int d^{2} \boldsymbol{k}_{1 \perp} d^{2} \boldsymbol{k}_{2 \perp} \delta^{2}\left(\boldsymbol{k}_{1 \perp}+\boldsymbol{k}_{2 \perp}-\boldsymbol{P}_{1 \perp}-\boldsymbol{P}_{2 \perp}\right) \\
\times & \left(\left(\boldsymbol{k}_{1 \perp} \cdot \boldsymbol{k}_{2 \perp}\right)\left(\boldsymbol{P}_{1 \perp} \cdot \boldsymbol{P}_{2 \perp}\right)-\left(\boldsymbol{k}_{1 \perp} \cdot \boldsymbol{P}_{1 \perp}\right)\left(\boldsymbol{k}_{2 \perp} \cdot \boldsymbol{P}_{2 \perp}\right)-\left(\boldsymbol{k}_{1 \perp} \cdot \boldsymbol{P}_{2 \perp}\right)\left(\boldsymbol{k}_{2 \perp} \cdot \boldsymbol{P}_{1 \perp}\right)\right) h_{1}^{\perp q}\left(x_{1}, \boldsymbol{k}_{1 \perp}^{2}\right) h_{1}^{\perp q}\left(x_{2}, \boldsymbol{k}_{2 \perp}^{2}\right),(13
\end{aligned}
$$

where $C_{q q}$ is the color factor which will be determined below. This result is very similar to the one obtained in photonjet production, and given in Ref. [30], where a $\cos 2 \phi$ angular dependence of photon-jet production is identified. Here we will use a weighting procedure on Eq. (13). The advantage is that the integrations over $\boldsymbol{k}_{1 \perp}$ and $\boldsymbol{k}_{2 \perp}$ can be deconvoluted. We find that using the weighting function $\mathcal{W}=\frac{P_{\perp}^{2} \cos \delta \phi}{M^{2}}$ and integrating the cross-section in Eq. (13) over $\boldsymbol{P}_{2 \perp}$, with $\delta \phi=\pi-\left(\phi_{2}-\phi_{1}\right)$, we can arrive at a simpler form:

$$
\begin{aligned}
& \frac{d \sigma^{\mathcal{W}}\left[J_{q} J_{q}\right]}{d \boldsymbol{P}_{\perp}^{2} d \eta_{1} d \eta_{2}}=\int d \phi_{1} d^{2} P_{2 \perp} \frac{P_{\perp}^{2} \cos \delta \phi}{M^{2}} \\
\times & \frac{d \sigma^{A}\left[J_{q} J_{q}\right]}{d^{2} \boldsymbol{P}_{1 \perp} d^{2} \boldsymbol{P}_{2 \perp} d \eta_{1} d \eta_{2}} \\
= & \frac{16 \pi C_{q q} \alpha_{s}^{2}}{s \boldsymbol{P}_{\perp}^{2}} y(1-y) \sum_{q} e_{q}^{2} h_{1}^{\perp,(1)}\left(x_{1}\right) h_{1}^{\perp,(1)}\left(x_{2}\right),
\end{aligned}
$$

where $h_{1}^{\perp(1)}(x)$ is the first $\boldsymbol{k}_{\perp}^{2}$-moment of $h_{1}^{\perp}\left(x, \mathbf{k}_{T}^{2}\right)$ :

$$
h_{1}^{\perp(1)}(x)=\int d^{2} \boldsymbol{k}_{\perp} \frac{\boldsymbol{k}_{\perp}^{2}}{2 M^{2}} h_{1}^{\perp}\left(x, \boldsymbol{k}_{\perp}^{2}\right)
$$


Eq. (14) shows that the angular dependent part of the di-jet production cross-section can be obtained by a cos $\delta \phi$ moment of the total cross-section.

Now we will calculate the color factor $C_{q q}$ appearing in Eq. (14). There are two sources which contribute to $C_{q q}$. One is the color factor from the hard scattering subprocess. For the diagram in Fig. 11k, this color factor is $-\frac{N^{2}-1}{4 N^{3}}$, which is the standard result of the generalized parton model. The other source is the special factor due to the presence of $T$-odd distributions, the so call gluonic pole factor [19, 21]. The key ingredients corresponding to this factor are the initial- and final-state interactions (the gluon exchanges before and after the hard scattering), which lead to the gauge-links appearing in the definition of TMD T-odd distributions. The simplest example is that every $T$-odd distribution function will contribute a factor of +1 in SIDIS and -1 in Drell-Yan process, due to the finaland initial-state interactions, which correspond to the future- and past-pointing gauge links, respectively. In the case of hadron scattering the situation is more involved, since the initial- and final- state interactions are mixed, which makes the determination of gluonic factors not straightforward. Systematic study of the gauge-links appearing in hadron scattering has been performed in Refs. [19, 21]. Here we will follow the approach presented in Refs. [19, 21] to calculate the gluonic pole factor coming from the product of two $h_{1}^{\perp}$. The general quark correlator with gauge link $\mathcal{U}$ is given by:

$$
\begin{aligned}
\Phi^{[\mathcal{U}]}\left(x, \boldsymbol{k}_{\perp}\right) & =\int d \xi^{-} \int \frac{d^{2} \boldsymbol{\xi}_{\perp}}{(2 \pi)^{2}} e^{i p \cdot \xi} \\
& \times\langle P S|\bar{\psi}(0) \mathcal{U}(0, \xi) \psi(\xi)| P S\rangle
\end{aligned}
$$

This gauge-link is process dependent and also depends on the hard subprocess. As we mentioned before, the subprocess shown in Fig. 1 can couple with $h_{1}^{\perp}$ and contributes to the angular dependence of di-jet in hadron scattering. The gauge link for the quarks which participate in this hard partonic process (the interference of $t$ and $u$ channel) can be written as [19]:

$$
\begin{aligned}
\mathcal{U}_{q q}^{\left[t u^{*}\right]}=\mathcal{U}_{q q}^{\left[t u^{*}\right]}= & \frac{2 N^{2}}{N^{2}-1} \frac{\operatorname{Tr}\left(\mathcal{U}^{[\square]}\right)}{N} \mathcal{U}^{[+]} \\
& -\frac{N^{2}+1}{N^{2}-1} \mathcal{U}^{[\square]} \mathcal{U}^{[+]}
\end{aligned}
$$

where

$$
\mathcal{U}^{[\square]}=\mathcal{U}^{[-] \dagger} \mathcal{U}^{[+]}
$$

is the gauge link loop, and $\mathcal{U}^{[+]}$and $\mathcal{U}^{[-]}$are the path-ordered future and past pointing Wilson lines. The two terms appearing in Eq. (17) correspond to the contributions from different color flows of the $q q \rightarrow q q$ process in the $t u^{*}$ channel. The convolution of the soft parts in the decomposition of two color flows is therefore

$$
\begin{aligned}
\Phi^{[\mathcal{U}]} \otimes \Phi^{\left[\mathcal{U}^{\dagger}\right]} \sim & \frac{2 N^{2}}{N^{2}-1} \Phi^{[(\square)+]} \otimes \Phi^{\left[(\square)++^{\dagger}\right]} \\
& -\frac{N^{2}+1}{N^{2}-1} \Phi^{[\square+]} \otimes \Phi^{\left[\square++^{\dagger}\right]}
\end{aligned}
$$

where $(\square)+$ and $\square+$ are the shortcuts of the basic gauge links $\frac{\operatorname{Tr}\left(\mathcal{U}^{[\square]}\right)}{N} \mathcal{U}^{[+]}$and $\mathcal{U}^{[\square]} \mathcal{U}^{[+]}$, respectively.

Here we will consider only the T-odd part of the contribution, the so call gluonic-pole contribution, since we are interested in the contribution of products of two $h_{1}^{\perp}$. As shown in Ref. [19, 21], after weighting by the transverse momentum, the $T$-odd part of each basic soft correlator will appear as the T-odd distribution function (in our case the T-odd functions is $\left.h_{1}^{\perp(1)}\right)$ multiplied by a prefactor. For example, the correlator $\Phi^{[(\square)+]}$ will give a factor 1 , while $\Phi^{[\square+]}$ will contribute a factor 3 . Therefore, the factor contributed by the product of two $h_{1}^{\perp}$ is:

$$
\begin{aligned}
& \left(\frac{2 N^{2}}{N^{2}-1} \times 1 \times 1-\frac{N^{2}+1}{N^{2}-1} \times 3 \times 3\right) \\
= & -\frac{7 N^{2}+9}{N^{2}-1} .
\end{aligned}
$$

We emphasize that this factor is the result coming from the $T$-odd distributions due to their complicated gauge-link structure, which cannot be predicted by the generalized parton model. After considering the color factor from the 
hard process, we get $C_{q q}$ as:

$$
\begin{aligned}
C_{q q} & =-\frac{N^{2}-1}{4 N^{3}} \times\left(-\frac{7 N^{2}+9}{N^{2}-1}\right) \\
& =\frac{7 N^{2}+9}{4 N^{3}} .
\end{aligned}
$$

Therefore the final result of the $\cos \delta \phi$-moment shown in Eq. (14) is

$$
\begin{aligned}
\frac{d \sigma^{W}\left[J_{q} J_{q}\right]}{d P_{\perp}^{2} d \eta_{1} d \eta_{2}} & =\frac{4 \pi \alpha_{s}^{2}}{s \boldsymbol{P}_{\perp}^{2}} \frac{7 N^{2}+9}{N^{3}} y(1-y) \\
& \times \sum_{q} h_{1}^{\perp(1) q}\left(x_{1}\right) h_{1}^{\perp(1) q}\left(x_{2}\right) .
\end{aligned}
$$

We then can define a subprocess $\cos \delta \phi$ asymmetry in $H_{A}+H_{B} \rightarrow J_{q}+J_{q}+X$ processes as

$$
R_{q q}=\frac{d \sigma^{\mathcal{W}}\left[J_{q} J_{q}\right] / d P_{\perp}^{2} d \eta_{1} d \eta_{2}}{d \sigma^{O}\left[J_{q} J_{q}\right] / d P_{\perp}^{2} d \eta_{1} d \eta_{2}}=\frac{4\left(7 N^{2}+9\right) \sum_{q} h_{1}^{\perp(1) q}\left(x_{1}\right) h_{1}^{\perp(1) q}\left(x_{2}\right)}{\left\{\frac{N^{3}-N}{2}\left(\frac{1+(1-y)^{2}}{y^{2}}+\frac{1+y^{2}}{(1-y)^{2}}\right)-\left(N^{2}-1\right) \frac{1}{y(1-y)}\right\} \sum_{q} f_{1}^{q}\left(x_{1}\right) f_{1}^{q}\left(x_{2}\right)}
$$

The main result given by this asymmetry is that there is an additional factor $-\frac{7 N^{2}+9}{N^{2}-1}$, as shown in (20), apart from the one obtained in the standard generalized parton model. The size of this gluonic pole factor is -9 for $N=3$, which is quite large and will greatly enhance the asymmetry. Moreover, comparing to the result from generalized parton model, the sign of the asymmetry is reversed by this gluonic pole factor.

\section{B. Process $H_{A}+H_{B} \rightarrow J_{q}+J_{\bar{q}}+X$}

Now we will consider the process $H_{A}+H_{B} \rightarrow J_{q}+J_{\bar{q}}+X$, where the partonic subprocesses contributing include $q \bar{q} \rightarrow q \bar{q}$ and $g g \rightarrow q \bar{q}$, as shown in Figs. 2 and 3, respectively. The diagram in 2a is also present for $q \bar{q} \rightarrow q^{\prime} \bar{q}^{\prime}$, which will contribute to $J_{q}+J_{\bar{q}}$ di-jet production. As before, the angular independent cross-section of $H_{A}+H_{B} \rightarrow J_{q}+J_{\bar{q}}+X$ process can be readily written as

$$
\begin{aligned}
\frac{d \sigma^{O}\left[J_{q} J_{\bar{q}}\right]}{d^{2} \boldsymbol{P}_{1 \perp} d^{2} \boldsymbol{P}_{2 \perp} d \eta_{1} d \eta_{2}}= & \frac{\alpha_{s}^{2}}{s \hat{s}} \int d^{2} \boldsymbol{k}_{1 \perp} d^{2} \boldsymbol{k}_{2 \perp} \delta^{2}\left(\boldsymbol{k}_{1 \perp}+\boldsymbol{k}_{2 \perp}-\boldsymbol{P}_{1 \perp}-\boldsymbol{P}_{2 \perp}\right)\left\{\left(\frac{N^{2}-1}{N^{2}}\left(\frac{1+(1-y)^{2}}{2 y^{2}}+(1-y)^{2}+y^{2}\right)\right.\right. \\
& \left.+\frac{\left(N^{2}-1\right)}{N^{3}} \frac{(1-y)^{2}}{y}\right) \sum_{q}\left(f_{1}^{q}\left(x_{1}, \boldsymbol{k}_{1 \perp}^{2}\right) f_{1}^{\bar{q}}\left(x_{2}, \boldsymbol{k}_{2 \perp}^{2}\right)+\left(x_{1} \leftrightarrow x_{2}\right)\right) \\
& \left.+\left(\frac{1}{2 N} \frac{y^{2}+(1-y)^{2}}{y(1-y)}-\frac{N}{N^{2}-1} y^{2}(1-y)^{2}\right) f_{1}^{g}\left(x_{1}, \boldsymbol{k}_{1 \perp}^{2}\right) f_{1}^{g}\left(x_{2}, \boldsymbol{k}_{2 \perp}^{2}\right)\right\}
\end{aligned}
$$

and the corresponding $\boldsymbol{P}_{2 \perp}$-integrated cross-section is given by

$$
\begin{aligned}
\frac{d \sigma^{O}\left[J_{q} J_{\bar{q}}\right]}{d P_{\perp}^{2} d \eta_{1} d \eta_{2}}= & \frac{\pi \alpha_{s}^{2}}{s \hat{s}}\left\{( \frac { N ^ { 2 } - 1 } { N ^ { 2 } } ( \frac { 1 + ( 1 - y ) ^ { 2 } } { 2 y ^ { 2 } } + 1 - 2 y + 2 y ^ { 2 } ) + \frac { ( N ^ { 2 } - 1 ) } { N ^ { 3 } } \frac { ( 1 - y ) ^ { 2 } } { y } ) \sum _ { q } \left(f_{1}^{q}\left(x_{1}\right) f_{1}^{\bar{q}}\left(x_{2}\right)\right.\right. \\
& \left.\left.+\left(x_{1} \leftrightarrow x_{2}\right)\right)+\left(\frac{1}{2 N} \frac{y^{2}+(1-y)^{2}}{y(1-y)}-\frac{N}{N^{2}-1} y(1-y)\right) f_{1}^{g}\left(x_{1}\right) f_{1}^{g}\left(x_{2}\right)\right\}
\end{aligned}
$$

Again, since $h_{1}^{\perp}$ is a chiral-odd function which flips the helicity of the initial and final partons, the hard diagrams that can contribute to the angular asymmetry in $H_{A}+H_{B} \rightarrow J_{q}+J_{\bar{q}}$ are given in Fig. 2a, Fig. 20 and Fig. 2d. Following a similar procedure as that used in the previous subsection, we can give the $\cos \delta \phi$-moment of $H_{A}+H_{B} \rightarrow J_{q}+J_{\bar{q}}$ 

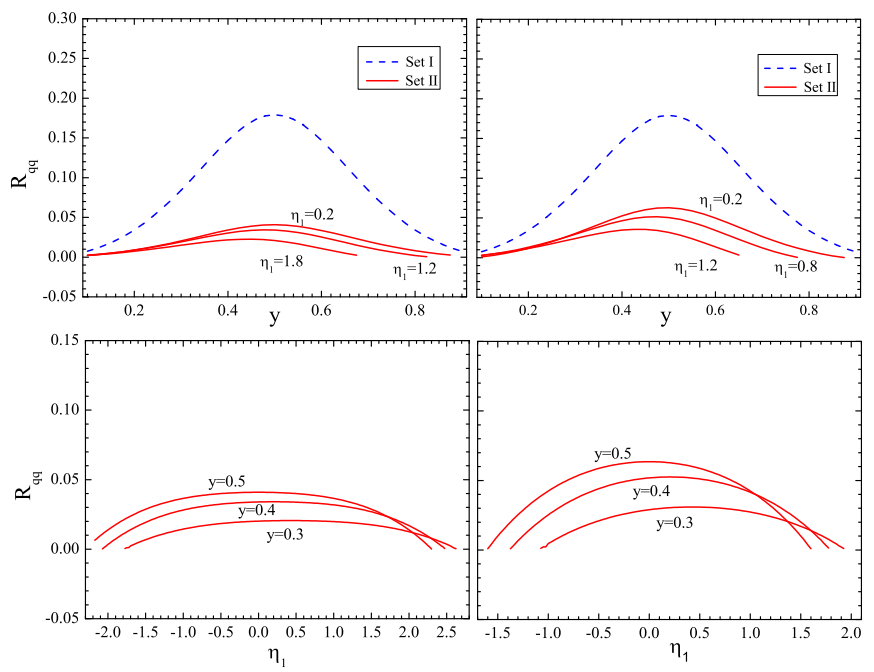

FIG. 4: The subprocess $\cos \delta \phi$ asymmetry $R_{q q}$ in di-jet production $H_{A}+H_{B} \rightarrow J_{q}+J_{q}+X$ at $\sqrt{s}=200 \mathrm{GeV}$, for $P_{\perp}=10 \mathrm{GeV}$ (left column) and $20 \mathrm{GeV}$ (right column). The dashed line and solid lines are the results from taking Boer-Mulders functions of Set I and II, respectively. The upper and lower panels show the asymmetry vs $y$ and $\eta_{1}$, respectively.

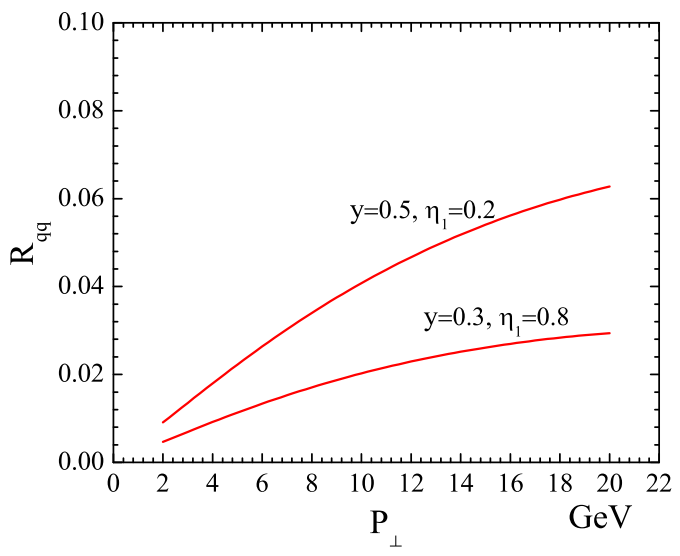

FIG. 5: The $P_{\perp}$-dependent subprocess $\cos \delta \phi$ asymmetry $R_{q q}$ in di-jet production $H_{A}+H_{B} \rightarrow J_{q}+J_{q}+X$ at $\sqrt{s}=200 \mathrm{GeV}$ calculated from the Boer-Mulders functions in Set II.

using the weighting function $\mathcal{W}=\frac{P_{\perp}^{2} \cos \delta \phi}{M^{2}}$, as follows

$$
\begin{aligned}
\frac{d \sigma^{\mathcal{W}}\left[J_{q} J_{\bar{q}}\right]}{d P_{\perp}^{2} d \eta_{1} d \eta_{2}} & =\int d \phi_{1} d^{2} \boldsymbol{P}_{2 \perp} \mathcal{W} \frac{d \sigma^{A}\left[J_{q} J_{\bar{q}}\right]}{d^{2} \boldsymbol{P}_{1 \perp} d^{2} \boldsymbol{P}_{2 \perp} d \eta_{1} d \eta_{2}} \\
& =\frac{16 \pi \alpha_{s}^{2}}{s P_{\perp}^{2}}\left(C_{q \bar{q}} y^{2}(1-y)^{2}+C_{q \bar{q}}^{\prime} y^{2}(1-y)\right) \\
& \times \sum_{q}\left(h_{1}^{\perp,(1), q}\left(x_{1}\right) h_{1}^{\perp,(1), \bar{q}}\left(x_{2}\right)+\left(x_{1} \leftrightarrow x_{2}\right)\right),
\end{aligned}
$$

The factors $C_{q \bar{q}}$ and $C_{q \bar{q}}^{\prime}$ can be calculated by combining the contribution of the hard subprocess and the gluonic pole factor. The term with $C_{q \bar{q}}$ is the result coming from the hard process in Fig. 22a, and the color factor of this process is $\frac{N^{2}-1}{4 N^{2}}$. The term with $C_{q \bar{q}}^{\prime}$ is the result from the hard processes Fig. $22 \mathrm{k}$ and Fig. $2 \mathrm{~d}$. The color factor of these processes is $-\frac{N^{2}-1}{4 N^{3}}$. The gluonic pole factors for those processes can be calculated from the corresponding gauge-links. For the 
diagrams in Fig. 2an, c and d, the gauge-links are equivalent, and given by:

$$
\begin{aligned}
\mathcal{U}_{q \bar{q}}^{\left[s \bar{q}^{*}\right]}= & \mathcal{U}_{q \bar{q}}^{\left[s \bar{q}^{*}\right]}=\mathcal{U}_{q \bar{q}}^{\left[t s^{*}\right]}=\frac{N^{2}}{N^{2}-1} \Phi^{[(\square)+]} \\
& -\frac{1}{N^{2}-1} \Phi^{[-]} .
\end{aligned}
$$

The convolution of the soft parts in the decomposition of two color flows is therefore

$$
\begin{aligned}
\Phi^{[\mathcal{U}]} \otimes \Phi^{\left[\mathcal{U}^{\dagger}\right]} \sim & \frac{2 N^{2}}{N^{2}-1} \Phi^{[(\square)+]} \otimes \Phi^{\left[(\square)+^{\dagger}\right]} \\
& -\frac{1}{N^{2}-1} \Phi^{[-]} \otimes \Phi^{\left[-^{\dagger}\right]}
\end{aligned}
$$

Therefore the factor contributed by the product of $h_{1}^{\perp}$ and $\bar{h}_{1}^{\perp}$ is calculated as:

$$
\frac{N^{2}}{N^{2}-1} \times 1 \times 1-\frac{1}{N^{2}-1} \times(-1) \times(-1)=1 .
$$

Thus we conclude that $C_{q \bar{q}}=\frac{N^{2}-1}{4 N^{2}}$ and $C_{q \bar{q}}^{\prime}=-\frac{N^{2}-1}{4 N^{3}}$. Unlike in the case of $J_{q}+J_{q}$ production, this result shows that in the case of $J_{q}+J_{\bar{q}}$ production there is no color factor enhancement for the azimuthal angle dependent cross-section, being therefore equivalent to the generalized parton model result. Thus we can give the subprocess $\cos \delta \phi$ asymmetry of $J_{q}+J_{\bar{q}}$ as the ratio of Eq. (26) and (25):

$$
\begin{aligned}
& R_{q \bar{q}}=\frac{d \sigma^{\mathcal{W}}\left[J_{q} J_{\bar{q}}\right] / d P_{\perp}^{2} d \eta_{1} d \eta_{2}}{d \sigma^{O}\left[J_{q} J_{\bar{q}}\right] / d P_{\perp}^{2} d \eta_{1} d \eta_{2}}= \\
& \frac{A(y) \sum_{q}\left(h_{1}^{\perp,(1), q}\left(x_{1}\right) h_{1}^{\perp,(1), \bar{q}}\left(x_{2}\right)+\left(x_{1} \leftrightarrow x_{2}\right)\right)}{B(y) \sum_{q}\left(f_{1}^{q}\left(x_{1}\right) f_{1}^{\bar{q}}\left(x_{2}\right)+\left(x_{1} \leftrightarrow x_{2}\right)\right)+C(y) f_{1}^{g}\left(x_{1}\right) f_{1}^{g}\left(x_{2}\right)},
\end{aligned}
$$

where

$$
\begin{aligned}
A(y)= & 4\left(y(1-y)-\frac{y}{N}\right), \\
B(y)= & \frac{1+(1-y)^{2}}{2 y^{2}}+(1-y)^{2}+y^{2} \\
& +\frac{1}{N} \frac{(1-y)^{2}}{y}, \\
C(y)= & \left(\frac{N^{2}-1}{2 N^{3}} \frac{y^{2}+(1-y)^{2}}{y(1-y)}-\frac{1}{N} y(1-y)\right) .
\end{aligned}
$$

\section{NUMERICAL RESULTS}

In the previous section we have calculated the theoretical result for the azimuthal angle dependence of di-jet production in unpolarized hadron scattering, and we showed that the azimuthal angle dependent part of the crosssection can be separated from the azimuthal angle independent part by taking a $\cos \delta \phi$-moment. We defined the $\cos \delta \phi$ asymmetry as the ratio of $\cos \delta \phi$-moment and the azimuthal angle independent cross-section. It is interesting to study wether the asymmetry could be accessed in hadron colliders. The Relativistic Heavy Ion Collider (RHIC) at BNL is running the polarized di-jet production process $p^{\uparrow}+p \rightarrow J_{1}+J_{2}+X$ [33], with the main purpose of measuring the transverse single spin asymmetry. The process can also be used to access the unpolarized di-jet production by averaging the spin-dependent data. We therefore calculate the subprocess asymmetry $R_{q q}$ given in Eq. (23) at RHIC energy $(\sqrt{s}=200 \mathrm{GeV})$, and show the result in Fig. 4. In the calculation we take $N=3$ and apply two sets of Boer-Mulders functions. Set I is the Boer-Mulders functions which saturate the positivity bound:

$$
h_{1}^{\perp(1) q}(x) \leq \frac{\left\langle\left|\boldsymbol{k}_{\perp}\right|\right\rangle}{2 M} f_{1}^{q}(x),
$$

with $\left\langle\left|\boldsymbol{k}_{\perp}\right|\right\rangle=0.44 \mathrm{GeV}$ [34]. Therefore this Set Boer-Mulders function will give the maximum bound of the asymmetries. In Set II we apply the Boer-Mulders functions which are extracted in Ref. [35] from unpolarized $p+D$ Drell-Yan 


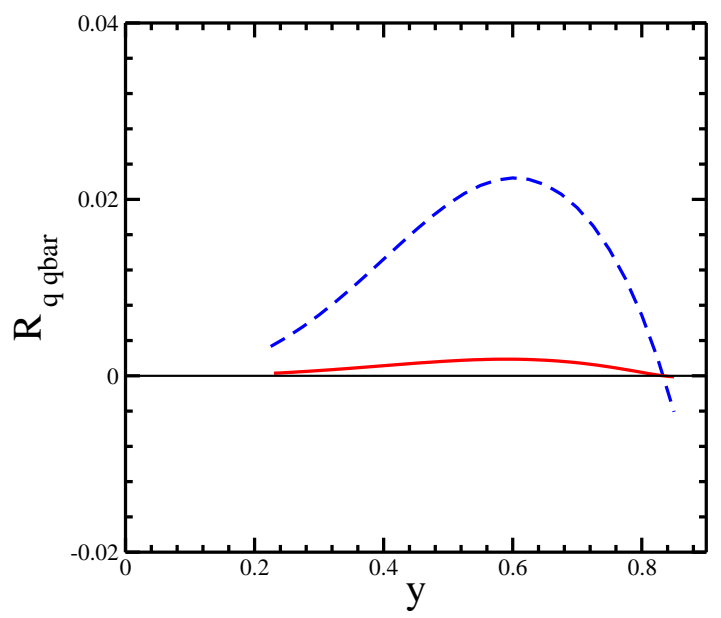

FIG. 6: The subprocess $\cos \delta \phi$ asymmetry $R_{q \bar{q}}$ in di-jet production $H_{A}+H_{B} \rightarrow J_{q}+J_{\bar{q}}+X$ at $\sqrt{s}=200 \mathrm{GeV}$ for $P_{\perp}=20$ $\mathrm{GeV}$. The dashed line and solid lines are the results from Boer-Mulders functions of Set I and II, respectively.

data [9] measured by the FNAL-E866/NuSea Collaboration. As shown in that paper, the Boer-Mulders functions in Set II can successfully reproduce the $\cos 2 \phi$ asymmetry in the unpolarized $p+D$ Drell-Yan process. So far the information on these functions is limited, therefore we adopt these two sets of Boer-Mulders functions to perform estimates on the $\cos \delta \phi$ asymmetries. For the unpolarized parton distributions appearing in the denominators of Eqs. (23) and (30) adopt the MRST2001 (LO set) parametrization [36]. The results for $R_{q q}$ from sets I and II are plotted with dashed and solid lines, respectively. The upper panel of Fig. 4 shows the subprocess asymmetries vs $y$ at $P_{\perp}=10 \mathrm{GeV}$ (left) and $P_{\perp}=20 \mathrm{GeV}$ (right) respectively, while the lower panel shows the subprocess asymmetries vs $\eta_{1}$ at same $P_{\perp}$. In Fig. 5 we also show $R_{q q}$ vs $P_{\perp}$ which is calculated from the Boer-Mulders functions in Set II. The result from Set I, which can be understood as the upper limit of the asymmetry, can be more than $10 \%$ in size. The result from Set II is about several percent. which is still quite sizable. The main reason of this result is the enhancement factor of -9 on the asymmetry from T-odd distributions in hadron scattering.

We also calculate the subprocess $\cos \delta \phi$ asymmetry of $J_{q}+J_{\bar{q}}$ production given in Eq. (30) at $\sqrt{s}=200 \mathrm{GeV}$, and show $y$-dependent curves at $P_{\perp}=20 \mathrm{GeV}$ and $\eta_{1}=0.2$ in Fig. 6. Our calculation indicates a subprocess smaller asymmetry for $J_{q}+J_{\bar{q}}$ production than that for $J_{q}+J_{q}$ production. This can be understood from the fact that there is no color factor enhancement for the azimuthal angle dependent cross-section of $J_{q}+J_{\bar{q}}$ production, and there is a large gluon-gluon scattering contribution in the denominator of Eq. (30).

In a practical measurement of di-jet production, the flavor of the jets usually is not measured. Hence the subprocess asymmetries shown in Figs. 4, 5and 6 are not measurable. The purpose to present those figures is to show how much the contribution coming from each subprocess is. Therefore we calculate the $\cos \delta \phi$ asymmetry of any di-jet production $R_{J J}$, which is measurable and defined as

$$
R_{J J}=\frac{\sum_{a, b=q, \bar{q}, g} d \sigma^{\mathcal{W}}\left[J_{a} J_{b}\right] / d P_{\perp}^{2} d \eta_{1} d \eta_{2}}{\sum_{a, b=q, \bar{q}, g} d \sigma^{O}\left[J_{a} J_{b}\right] / d P_{\perp}^{2} d \eta_{1} d \eta_{2}}
$$

In this paper we assume that the partonic processes that contribute to the azimuthal angular dependence of di-jet production are $q q \rightarrow q q$ and $q \bar{q} \rightarrow q \bar{q}$. Therefore the numerator in Eq. (35) is the sum of Eqs. (22) and (26), while the denominator contains the contributions from Eqs. (12), (25) and from all other possible processes, such as $q q^{\prime} \rightarrow q q^{\prime}$, $q g \rightarrow q g$, and so on. In Fig. 7 we show by thin lines the asymmetry $R_{J J}$ at RHIC for $P_{\perp}=20 \mathrm{GeV}$ vs $y$, from the two sets of Boer-Mulders functions (solid and dashed lines for Set I and Set II respectively). As a comparison, we also show by thick lines the result of $R_{J J}$ from the conventional generalized parton model (GPM) calculation where gluonic pole contributions are not considered. In Fig. 7b, we show the asymmetry $R_{J J}$ at RHIC for $P_{\perp}=10 \mathrm{GeV}$ (thick lines) and $P_{\perp}=20 \mathrm{GeV}$ (thin lines) vs $\eta_{1}+\eta_{2}$. In the calculations we restrict $-1<\eta_{1 / 2}<2$ to consider the pseudorapidity acceptance of jet at RHIC. The curves given in Fig. $7 \mathrm{~g}$ indicate a sign reversal of the asymmetries $R_{J J}$ between the results from the two different approaches. This is due to the additional factor -9 in the azimuthal angular dependent part of $H_{A}+H_{B} \rightarrow J_{q}+J_{q}+X$, coming from the multiple initial- and final-state interactions. We remind that a similar sign reversal also happens in the SSA in hadronic photon-jet production [27], which is due to a similar effect. Therefore the measurement of the $\cos \delta \phi$ asymmetry $R_{J J}$ in unpolarized di-jet production at RHIC, especially the enhancement of the size and the sign reverse of the asymmetry, will provide further tests on the role 

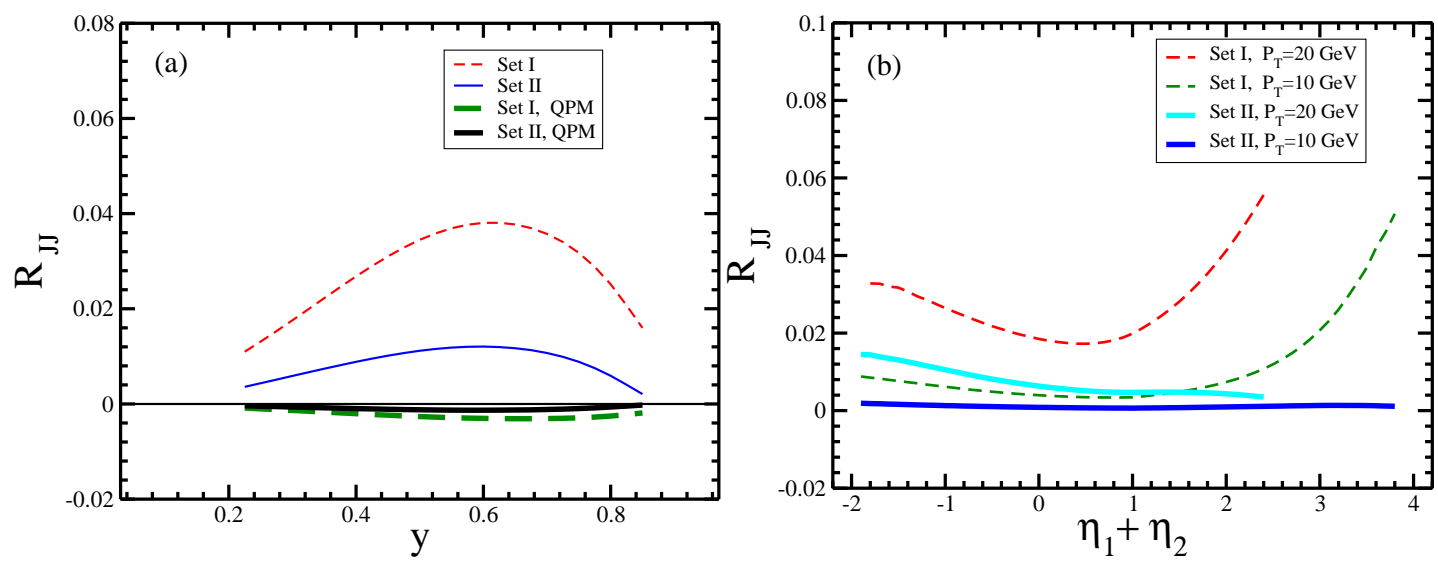

FIG. 7: (a), The $y$-dependent $\cos \delta \phi$ asymmetry $R_{J J}$ in di-jet production $H_{A}+H_{B} \rightarrow J_{1}+J_{2}+X$ at RHIC for $P_{\perp}=20$ GeV. The dashed line and solid lines are the results from Boer-Mulders functions of Set I and II, respectively. We also show by thick lines the result of $R_{J J}$ from the conventional generalized parton model (GPM) calculation where gluonic pole contributions are not considered. (b), The $\cos \delta \phi$ asymmetry $R_{J J}$ vs $\eta_{1}+\eta_{2}$ in di-jet production $H_{A}+H_{B} \rightarrow J_{1}+J_{2}+X$ at RHIC for $P_{\perp}=10$ $\mathrm{GeV}$ (thick lines) and $P_{\perp}=20 \mathrm{GeV}$ (thin lines) from Boer-Mulders functions of Set I (thin lines) and II (thick lines).

of initial- and final-state interactions as QCD dynamics of $T$-odd distributions in hadron scattering. We would like point out that in calculating $R_{J J}$, we only considered the lowest order contributions to parton scattering. Other contributions may affect the size of the asymmetry, such as NLO corrections for the denominator of Eq. (35). At large $P_{\perp}$ they are further enhanced by threshold logarithms which are more pronounced for gluonic channels. Therefore our results are estimates and $R_{J J}$ can be smaller. However we expect that those contributions will not change the sign of the asymmetry.

\section{SUMMARY}

In summary, we performed a study of the azimuthal angular dependence of back-to-back di-jet production in unpolarized hadron scattering, focusing our attention on the process $H_{A}+H_{B} \rightarrow J_{q}+J_{q}+X$, where two quark jets have the same flavor, and on the process $H_{A}+H_{B} \rightarrow J_{q}+J_{\bar{q}}+X$, where the di-jet comes from a quark-antiquark pair. We find that in both processes there are an azimuthal angle dependent cross-section generated by the product of two $h_{1}^{\perp}$ from each incident hadron. Using a weighting function $\cos \delta \phi$, we can separate the azimuthal angle dependent cross-section from the azimuthal angle independent one. We defined the $\cos \delta \phi$ asymmetry of di-jet production as the ratio between the $\cos \delta \phi$-moment and the azimuthal angle independent cross-section. In the case of $J_{q}+J_{q}$ production, due to the multiple initial- and final-state interactions within hadron scattering, there is a color factor enhancement (which is -9 for $N=3$ ) in the asymmetry compared to the result from the standard generalized parton model. No such enhancement appears in the case of $J_{q}+J_{\bar{q}}$ production. We estimate the $\cos \delta \phi$ asymmetry of di-jet production at the RHIC energy, and find that the subprocess asymmetry of $J_{q}+J_{q}$ production can reach up to $10 \%$ in maximum due to the large color factor enhancement. In contrast, the subprocess asymmetry of $J_{q}+J_{\bar{q}}$ production is smaller. We further find that the color factor enhancement in $J_{q}+J_{q}$ production can increase the size and reverse the sign of the total $\cos \delta \phi$ asymmetry $R_{J J}$ in di-jet production. Therefore it is feasible to perform the measurement on the di-jet production in unpolarized hadron scattering at RHIC, which can identify the color factor enhancement on the $\cos \delta \phi$ asymmetry in $J_{q}+J_{q}$ production. Furthermore, the study will provide the opportunity for a better understanding on the role of initial- and final-state interactions in hadron scattering and other processes.

Acknowledgements This work is supported by the PBCT project ACT-028 "Center of Subatomic Physics".

[1] P.J. Mulders and R.D. Tangerman, Nucl. Phys. B461, 197 (1996).

[2] D. Boer and P.J. Mulders, Phys. Rev. D57, 5780 (1998).

[3] A. Airapetian et al. (HERMES Collaboration), Phys. Rev. Lett. 94, 012002 (2005). 
[4] V.Yu. Alexakhin et al. (COMPASS Collaboration), Phys. Rev. Lett. 94, 202002 (2005).

[5] D.L. Adams et al. (E581 and E704 Collaboration), Phys. Lett. B261, 201 (1991); D.L. Adams et al. (E704 Collaboration), Phys. Lett. B264, 462 (1991).

[6] J. Adams et al. (STAR Collaboration), Phys. Rev. Lett. 92, 171801 (2004).

[7] S. Falciano et al. (NA10 Collaboration), Z. Phys. C 31, 513 (1986); M. Guanziroli et al. (NA10 Collaboration), Z. Phys. C 37, 545 (1988).

[8] J. S. Conway et al. (E615 Collaboration), Phys. Rev. D 39, 92 (1989).

[9] L.Y. Zhu, et. al., (FNAL-E866/NuSea Collaboration), Phys. Rev. Lett. 99, 082301 (2007).

[10] D. Sivers, Phys. Rev. D41, 83 (1990); Phys. Rev. D 43, 261 (1991).

[11] D. Boer, Phys. Rev. D 60, 014012 (1999).

[12] X. Ji and F. Yuan, Phys. Lett. B543, 66 (2002); A.V. Belitsky, X. Ji, and F. Yuan, Nucl. Phys. B656, 165 (2003).

[13] D. Boer, P.J. Mulders, and F. Pijlman, Nucl. Phys. B667, 201 (2003).

[14] J.C. Collins, Phys. Lett. B536, 43 (2002).

[15] S.J. Brodsky, D.S. Hwang, and I. Schmidt, Phys. Lett. B 530, 99 (2002); Nucl. Phys. B642, 344 (2002).

[16] J.C. Collins and A. Metz ,Phys. Rev. Lett. 93, 252001 (2004).

[17] X. Ji, J.P. Ma, and F. Yuan, Phys. Lett. B597, 299 (2004).

[18] C. J. Bomhof, P. J. Mulders, and F. Pijlman, Phys. Lett. B596, 277 (2004).

[19] A. Bacchetta, C. J. Bomhof, P. J. Mulders, and F. Pijlman, Phys. Rev. D 72, 034030 (2005).

[20] C. J. Bomhof, P. J. Mulders, and F. Pijlman, Eur. Phys. J. C 47, 147 (2006).

[21] C. J. Bomhof and P. J. Mulders, JHEP 0702, 029 (2007).

[22] J. W. Qiu , W. Vogelsang, and F. Yuan, Phys. Rev. D 76, 074029 (2007).

[23] W. Vogelsang and F. Yuan, Phys. Rev. D 76, 094013 (2007).

[24] D. Boer and W. Vogelsang, Phys. Rev. D 69, 094025 (2004).

[25] J. Collins and J. W. Qiu, Phys. Rev. D 75, 114014 (2007).

[26] C. J. Bomhof, P. J. Mulders, W. Vogelsang, and F. Yuan, Phys. Rev. D 75, 074019 (2007).

[27] A. Bacchetta, C. Bomhof, U. D'Alesio, P.J. Mulders, and F. Murgia, Phys. Rev. Lett. 99, 212002 (2007).

[28] John Collins, arXiv:0708.4410 [hep-ph].

[29] C. J. Bomhof and P. J. Mulders, Nucl. Phys. B795, 409 (2008).

[30] D. Boer, P.J. Mulders, and Pisano, Phys. Lett. B660, 360 (2008)

[31] D. Boer, S.J. Brodsky, and D.S. Hwang, Phys. Rev. D 67, 054003 (2003).

[32] Z. Lu and B.-Q. Ma, Phys. Lett. B615, 200 (2005).

[33] B. I. Abelev et.al., (STAR Collaboration), Phys. Rev. Lett. 99, 142003 (2007).

[34] M. Anselmino, M. Boglione, U. D'Alesio, A. Kotzinian, F. Murgia, and A. Prokudin, Phys. Rev. D 71, 074006 (2005).

[35] B. Zhang ,Z. Lu, B.-Q. Ma, and I. Schmidt, Phys. Rev. D 77, 054011 (2008).

[36] A. D. Martin, R. G. Roberts, W. J. Stirling, and R. S. Thorne, Phys. Lett. B 531, 216 (2002). 